\title{
CONTRIBUCIÓN DE LOS ECOSISTEMAS ALTOANDINOS EN LA PROVISIÓN DEL SERVICIO ECOSISTÉMICO DE REGULACIÓN HÍDRICA
}

\section{CONTRIBUTION OF HIGH-ANDEAN ECOSYSTEMS IN PROVIDING THE WATER REGULATION ECOSYSTEM SERVICE}

\author{
Ronal Cervantes ${ }^{1,2,3}$, José Miguel Sánchez ${ }^{1,4}$, Julio Alegre ${ }^{1,5}$, Eric Rendón ${ }^{1,6}$, Jan R. Baiker ${ }^{7}$, \\ Bruno Locatelli ${ }^{8}$ y Vivien Bonnesoeur ${ }^{9}$
}

\begin{abstract}
Resumen
El servicio ecosistémico de regulación hídrica es uno de los más importantes que ofrecen los ecosistemas altoandinos. Sin embargo, el conocimiento sobre su aporte en términos hídricos es aún escaso y su estimación difícil, debido a los complejos procesos eco-hidrológicos, climáticos y características ambientales de los Andes. Por ello, se estimó la influencia de tres tipos de ecosistemas (pajonal de puna húmeda, bofedal y bosque de Polylepis) sobre flujos de agua, particularmente los flujos que se dirigen hacia el almacenamiento subterráneo, que equivalen al servicio ecosistémico de regulación hídrica. Dicho estudio se desarrolló durante el año hidrológico 2018 - 2019 en la unidad hidrográfica de Rontoccocha, entre los 3900 a $4635 \mathrm{msnm}$, en el Departamento de Apurímac, Perú. Para ello se modeló el balance hídrico por cada tipo de ecosistema con la herramienta eco-hidrológica Hydrobal. Se usaron variables de: a) vegetación, b) parámetros climáticos y c) características del suelo. Los resultados revelan el aporte de la cobertura vegetal en la regulación hídrica. En cada ecosistema, cerca del 15\% de toda la lluvia que precipita en la cuenca logra llegar al almacenamiento subterráneo. Dichos datos extrapolados a toda la unidad hidrográfica, muestran que el pajonal de puna húmeda regula el $80 \%$, el bofedal el $17 \%$ y el bosque de Polylepis el 3\%. A pesar de que la evaluación se realizó por separado para cada ecosistema, para fines de gestión, es necesario abordarlo de manera integral dado que entre ellos se establecen relaciones de interdependencia.
\end{abstract}

Palabras clave: Andes, pajonal de puna húmeda, bofedal, bosque de Polylepis, balance hídrico.

\begin{abstract}
The ecosystem service of water regulation is one of the most important offered by the highAndean ecosystems. However, knowledge about its contribution in hydrological terms is still scarce and its estimation is difficult, due to the complex eco-hydrological and climatic processes, and environmental characteristics of the Andes. Therefore, the influence of three types of ecosystems (humid puna grassland, tropical high-Andean wetland (bofedal) and Polylepis forest) was estimated on water flows, particularly flows that reach underground storage, which is equivalent to the ecosystem service of water regulation. This study was developed during the hydrological year 2018 - 2019 in the Rontoccocha hydrographic unit, between 3900 and 4635 masl, in the Apurimac Region, Peru. For this, the water balance for each type of ecosystem was modeled with the Hydrobal eco-hydrological tool. Variables of: a) vegetation, b) climatic parameters, and c) soil characteristics were used. The results reveal the contribution of the vegetation cover in the hydric regulation. In each ecosystem, about $15 \%$ of all the rainfall that falls in the basin reaches underground storage. These data, extrapolated to the entire hydrographic unit, show that the humid puna grassland regulates $80 \%$, the tropical high-Andean wetland $17 \%$, and the Polylepis forest $3 \%$. Although the evaluation was carried out separately for each ecosystem, for management purposes, it is necessary to address them in an integrative way since interdependence relationships are established between them.
\end{abstract}

Key words: Andes, humid puna grassland, tropical high-Andean wetland, Polylepis forest, water balance.

\section{Introducción}

El servicio ecosistémico de regulación hídrica, entendida como la capacidad que tienen los ecosistemas para almacenar el agua en época de lluvia y liberarla en época seca (Llambí et al., 2012; MINAM 2019), es uno de los servicios más importantes para el bienestar humano, provistos por los ecosistemas altoandinos. Dichos ecosistemas de montaña proveen el agua de la cual dependen millones de personas (Buytaert et al., 2006; Bonnesoeur et al., 2019). Sin embargo, cambios en el uso del suelo y la cobertura vegetal se han convertido en los principales impulsores que alteran los servicios hidrológicos de estos ecosistemas (Ochoa-Tocachi et al., 2016). A nivel global, lo anterior pone en riesgo la seguridad hídrica de más del $50 \%$ de la humanidad que depende del agua 
Julio - Diciembre 2021

de las montañas para beber, irrigar y producir alimentos y energía (Viviroli et al., 2007). Así, en los ecosistemas altoandinos es importante entender su funcionamiento para promover su conservación y/o recuperación que permita garantizar la continua provisión de sus servicios ecosistémicos.

A pesar de que se reconoce la importancia de los ecosistemas en la regulación hídrica en la zona andina (sobre todo en zonas de puna y jalca), muy poco se ha estudiado acerca de su funcionamiento que permita entender su dinámica y cuantificar su aporte hídrico (Ochoa-Tocachi et al., 2016; Somers \& McKenzie, 2020). Dicha medición es un tema central para su gestión y diversos aspectos de política ambiental y de los recursos hídricos como: contabilidad ambiental, valoración de recursos naturales, prioridades de conservación y planificación (Brown et al., 2014).

En los Andes, medir el aporte de los ecosistemas en la regulación hídrica no es una tarea sencilla. OchoaTocachi et al. (2016) consideran que medir los servicios ecosistémicos, o los impactos sobre ella, está lleno de dificultades, fundamentalmente por la extrema diversidad y complejidad de la región andina: altos gradientes espaciales y temporales y la variabilidad en sus condiciones geográficas e hidrometeorológicas. En otros casos, no se valora adecuadamente la contribución del ecosistema en la regulación hídrica ya que, en la mayoría de los modelos de balance hídrico, se da menos peso a la vegetación y otros ni la consideran (Bellot \& Chirino, 2013; Touhami, 2014).

No es sorpresa que, ante los crecientes problemas de disponibilidad de agua, normalmente las inversiones prioricen la implementación de infraestructura física (gris), mientras que el aporte de los ecosistemas casi no son tomados en cuenta; sin embargo, cada vez más se reconoce el aporte de la naturaleza en la solución de los problemas de disponibilidad de agua y su rol complementario al enfoque tradicional de la infraestructura física; por lo tanto, las inversiones en infraestructura natural, no solo ayudarían a resolver los problemas de agua, sino también permitirían optimizar los recursos económicos debido a su bajo costo (León, 2016).

Para contribuir al cierre de las brechas mencionadas, el presente estudio busca responder a la incógnita del aporte de tres ecosistemas altoandinos (ubicados por encima de los $3900 \mathrm{msnm}$ ) al servicio de regulación hídrica: a) bofedales (humedales altoandinos), b) pradera de pajonal de puna húmeda, y c) bosques relictos de Polylepis ("queñoal"). Lo anterior brinda la oportunidad para resaltar el rol de los ecosistemas en la provisión de agua hacia las poblaciones ubicadas aguas abajo y entender mejor los temas de equidad en la gestión de los servicios ecosistémicos en una cuenca (Vallet et al., 2019; Vallet et al., 2020). Ello permitirá reorientar las prioridades de gestión ambiental en las cabeceras de cuenca, como las iniciativas de pago por servicios ecosistémicos hídricos (PSEH) o inversiones en infraestructura natural.

\section{Materiales y métodos}

El estudio se realizó en la unidad hidrográfica de Rontoccocha, ubicada en la cabecera de la cuenca del río Mariño, entre los 3900 a $4635 \mathrm{msnm}$, en Apurímac, Perú. Tiene un área de 875 ha y representa la segunda fuente más importante de agua de consumo humano para la ciudad de Abancay. En la zona se encuentran cuatro ecosistemas predominantes: 1) pajonal de puna húmeda $(66.9 \%) ; 2)$ bofedales $(13.8 \%)$; 3) bosque relicto de Polylepis (2.2\%) y 4) lagunas altoandinas (4.0\%), también existen afloramientos rocosos que ocupan el $13.1 \%$ (Figura 1).

El aporte de los ecosistemas a la regulación hídrica se realizó modelando el balance hídrico en el suelo con la herramienta eco-hidrológica Hydrobal, propuesto por Bellot \& Chirino (2013). Este es un modelo determinístico cuya ventaja, frente a otras, es que considera la vegetación como un factor importante en la regulación hídrica (Touhami, 2014). Hydrobal integra las condiciones meteorológicas, las características de la vegetación y los procesos del suelo a escala de parcela en ecosistemas dominados por diferentes tipos de vegetación. Con ello, las salidas permiten estimar la evapotranspiración real, interceptación, escorrentía superficial, el contenido de agua del suelo y el drenaje profundo para la recarga de acuíferos (Bellot \& Chirino, 2013). El modelo trabaja en función de la Fórmula 1.

$$
\Delta \mathrm{SWC}=\mathrm{P}-\text { Int }- \text { Eta }- \text { Roff }-\mathrm{Pc}
$$

\section{Fórmula 1.}

Donde:

$\triangle \mathrm{SWC}=$ contenido de agua en el suelo

$\mathrm{P}=$ Precipitación

Int $=$ Interceptación

Eta $=$ Evapotranspiración real

Roff = Escorrentía superficial

$\mathrm{Pc}=$ Percolación profunda

Obtención de datos

Se obtuvieron datos climáticos (precipitación y evapotranspiración potencial), datos de las características del suelo (textura, humedad, densidad aparente, capacidad de campo) y la vegetación (cobertura, composición y estructura de la vegetación).

Los datos de precipitación se obtuvieron de dos pluviómetros digitales (onset HOBO RG3-M; data logging rain gauge) instalados en la parte baja (4 062 msnm) y alta (4 $455 \mathrm{msnm}$ ) de la cuenca, que registraron información del período 2016-2020 (Figura 2); sin embargo, para el modelamiento solo se procesó los datos del año hidrológico 2018-2019. La evapotranspiración potencial se obtuvo del estudio hidrológico de la cuenca Mariño que procesa datos 
históricos del período 1965-2010, sobre la base de 14 estaciones del Servicio Nacional de Meteorología e Hidrología del Perú - SENAMHI (GRA, 2013).

Para la información de suelos se muestrearon seis puntos en pajonal, seis en bofedal y tres en bosque de Polylepis. De ellos se obtuvieron los datos de textura, porosidad, humedad y $\mathrm{pH}$. Esta primera muestra se complementó con datos del estudio edafológico del Proyecto Gestión Integral de la Microcuenca Mariño (GRA, 2013) y Proyecto de Recuperación de Ecosistemas Andinos de Bofedales en seis distritos de la provincia de Abancay y Grau, región Apurímac. Con ello, se llegó a un total de 14 muestras en bofedal, 15 en pajonal y cinco en bosque de Polylepis. Luego, con los datos previos y el programa "Soil Water Characteristics", del Departamento de Agricultura de los Estados unidos (USDA, 2016), se obtuvo la capacidad de campo (CC) y punto de marchitez permanente (PMP).

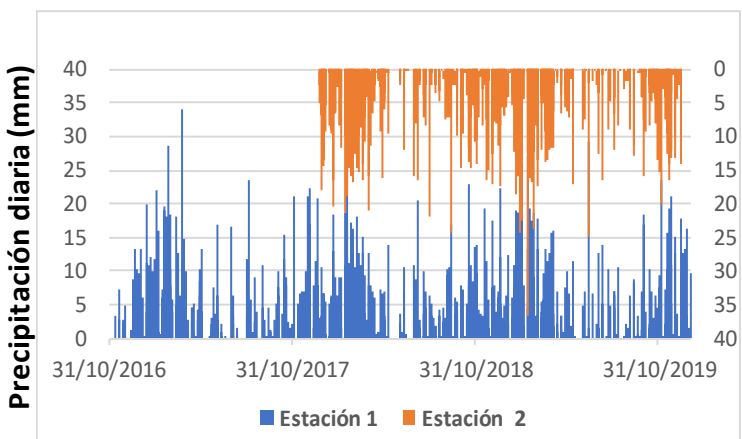

Estación 1: período 31/10/2016 - 02/01/2020, y estación 2: período 22/12/2017 - 13/12/2019.

Figura 2. Precipitación diaria en la unidad hidrográfica Rontoccocha.

Los datos de la vegetación se trabajaron en dos niveles: a) la cobertura en la unidad hidrográfica de Rontoccocha para conocer su distribución, y b) la composición y estructura de cada cobertura vegetal. La distribución de la cobertura vegetal se estableció mediante material cartográfico a escala 1:100 000 del proyecto "Validación y Publicación de la ZEE de la región Apurímac" (GRA, 2010), actualizado con imágenes satelitales Sentinel-2 del servidor Copernicus (ESA, 2018) (Figura 1). En tanto, la composición y estructura de los ecosistemas se analizó a nivel de las especies más representativas y/o dominantes, dado que para el propósito del estudio no se requiere el detalle taxonómico. Para ello, se establecieron 12 parcelas de observación de $1 \mathrm{~m}^{2}$ en los ecosistemas de pajonal y bofedal, respectivamente, y seis parcelas de $50 \mathrm{~m}^{2}$ en el bosque de Polylepis.

Análisis de datos

Con los datos descritos, con Hydrobal se calculó la evapotranspiración real, escorrentía, humedad del suelo diaria y drenaje profundo o recarga. Sin embargo, se hicieron ajustes en los resultados de escorrentía superficial de pajonal y bosque de Polylepis y de infiltración profunda en bofedal.

En el caso de escorrentía superficial, Hydrobal calcula dicho parámetro mediante una regresión lineal en función de la precipitación (Roff $=\mathrm{a}+\mathrm{b}(\mathrm{P})$; P es la precipitación diaria). Dado que la escorrentía también depende de otros factores como la pendiente, se incorporó dicho parámetro para mejorar el modelo en los ecosistemas de pajonal y bosque de Polylepis. Ese proceso se realizó mediante el método de Número de Curva, desarrollado por el "Soil Conservation Service" del Departamento de Agricultura de los Estados Unidos, adaptado por Sprenger (1978) para incluir la pendiente.

Para el caso bofedal, el ajuste se realizó debido a que se trata de un ecosistema pantanoso, cuyo nivel freático (profundidad de la napa freática) es observable. Por lo tanto, la capacidad de regulación de dicho ecosistema será la altura del nivel freático hasta la superficie del suelo; el excedente saldrá del sistema a manera de escorrentía, pues el suelo ya se encuentra saturado (USGS, 2016; Hasan \& Khan, 2019). Para ello, se midieron las profundidades máximas del nivel freático (altura mínima) en época seca, ya que a partir de ello comienza la regulación con la llegada de las lluvias. Dichos datos se obtuvieron de 37 puntos permanentes de observación: 20 registrados el 07/set/2019 y 15/oct/2019 y 17 puntos el 17/jul/2018, 19/set/2018 y 15/jun/2019. Para ello, se instalaron tubos de observación ("groundwater monitoring wells") que permiten apreciar la profundidad del nivel freático (Figura 3). El detalle de las variables insertadas en el modelo Hydrobal, se muestra en la Tabla 1.

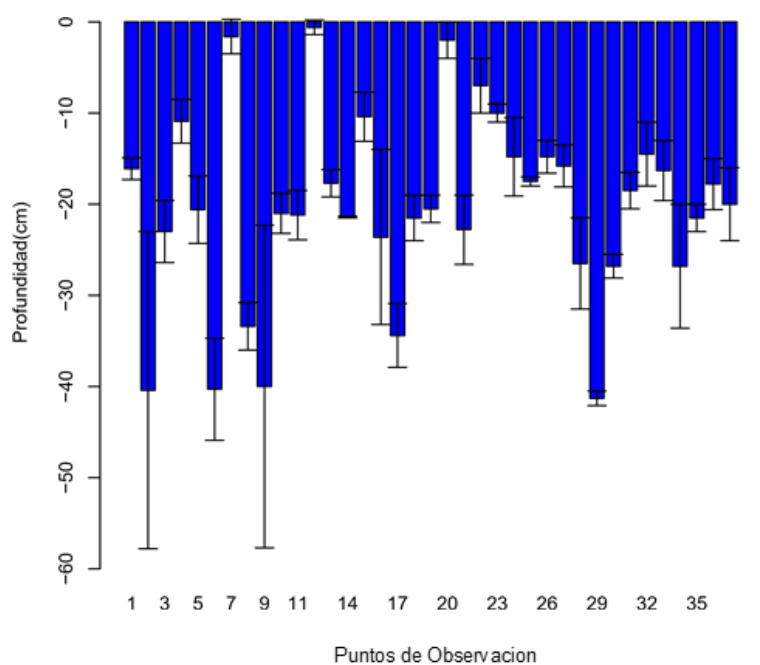

Figura 3. Profundidad media del nivel freático de 37 puntos de observación en época seca, en bofedales de la unidad hidrográfica de Rontoccocha. 
Julio - Diciembre 2021

Comprobación de los resultados

Para comprobar la validez de los resultados se hizo el balance hídrico en la laguna Rontoccocha que se encuentra en la parte baja de la microcuenca de estudio. Para ello, se asume que éste es el depósito final de los flujos superficiales y subterráneos de la unidad hidrográfica de Rontoccocha; por lo tanto, el volumen de caudal subterráneo calculado con la Fórmula 2 (Hayashi \& Van der Kamp, 2000; Lynn et al., 2006) debería coincidir con el estimado con Hydrobal.

$$
\Delta \mathrm{S}=\Sigma \mathrm{Q}_{\text {in }}+\mathrm{P}-\mathrm{E}-\mathrm{Q}_{\text {out }}+\mathrm{Q}_{\mathrm{Gwin}}-\mathrm{Q}_{\mathrm{Gwout}}
$$

\section{Fórmula 2.}

Donde:

$\Delta \mathrm{S}=$ Cambio en el almacenamiento de agua

$\Sigma Q_{\text {in }}=$ Suma de los ingresos de fuentes de agua superficial

$\mathrm{P}=$ Precipitación

$\mathrm{E}=$ Evaporación

$\mathrm{Q}_{\text {out }}=$ Salida de agua por fuentes superficiales

$\mathrm{Q}_{\mathrm{Gwin}}=$ Ingresos de agua subterránea

$\mathrm{Q}_{\mathrm{Gwout}}=$ Salidas de agua subterránea

En la laguna Rontoccocha, dado que la entrada y salida de agua subterránea no se midieron directamente, la Fórmula 1 se simplificó a la Fórmula 3.

$$
\Delta \mathrm{S}=\Sigma \mathrm{Q}_{\text {in }}+\mathrm{P}-\mathrm{E}-\mathrm{Q}_{\text {out }}+\mathrm{Q}_{\mathrm{Res}}
$$

Fórmula 3.

Donde QRes o agua residual subterránea es la cantidad neta de entrada y salida por flujo subterráneo (Lynn et al., 2006); por lo tanto, este valor debería ser aproximadamente igual al volumen calculado con Hydrobal: $Q_{\text {Res }} \cong$ Vol. calculado con Hydrobal (Figura 4). Para este propósito se usaron los caudales de ingreso y salida medidos en el estudio hidrológico de la cuenca Mariño (GRA, 2013) y la empresa prestadora de servicios de agua potable - Emusap Abancay. Para validar dichos datos, durante el 2018 y 2019 se hicieron mediciones de la altura de almacenamiento de agua en la laguna Rontoccocha, ingreso $y$ salida de agua superficial con una periodicidad de 15 días.

\section{Resultados y discusión}

Comportamiento de las principales variables ambientales en el ecosistema

Un primer aspecto que el estudio permitió analizar fue el comportamiento de las principales variables ambientales que influyeron en la regulación hídrica de los ecosistemas: precipitación neta, evapotranspiración real y escorrentía superficial.

Respecto a la precipitación neta, se observa que el bosque de Polylepis es el ecosistema con mayor nivel de interceptación (9.5\%) y, por lo tanto, menor precipitación neta en comparación con el bofedal y pajonal (Tabla 2). Debido a ello, menor agua llega al suelo para continuar con el ciclo hidrológico. Esta característica tiene sentido ya que el proceso de interceptación está determinado por las características estructurales del dosel, específicamente por el índice de área foliar (LAI por sus siglas en inglés) de la planta. Entonces, a mayor área foliar, mayor será la interceptación (Körner et al., 1989; Bellot \& Chirino, 2013; Valencia \& Tobón, 2017). Ello explica el por qué un bosque tiene mayor capacidad de interceptar la lluvia, que una cobertura de tipo graminoide como el pajonal y bofedal.

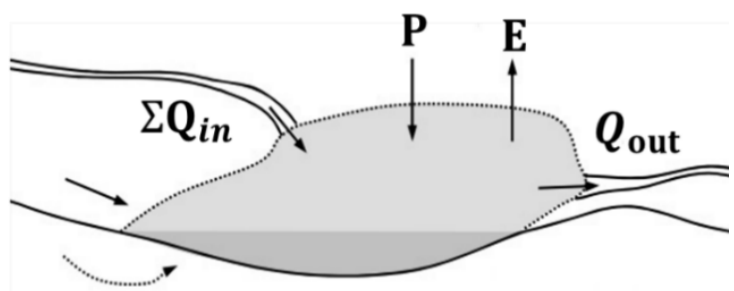

\section{ModeloHydrobal $\cong Q_{\text {Res }}$}

Adaptado de Hayashi \& Van der Kamp (2000).

Figura 4. Modelo de balance hídrico para comprobar la validez de los resultados de Hydrobal; donde la estimación con Hydrobal $\cong \mathrm{Q}_{\text {Res. }}$.

Respecto a la evapotranspiración real, los resultados de pajonal y bosque de Polylepis tienen comportamientos similares con 698 mm/año y 696 mm/año, respectivamente; sin embargo, en el ecosistema del bofedal la evapotraspiración llega a $765 \mathrm{~mm} /$ año (Tabla 2). Esta característica se debería a que el bofedal se desarrolla sobre un lecho húmedo que le permite obtener agua de forma permanente para su desarrollo fisiológico (Hayashi \& Van der Kamp, 2000; USGS, 2016). Así mismo, se aprecia que ésta es la principal vía de pérdida de agua del ecosistema (entre el 54 y $59 \%$ en promedio), con mayor intensidad en época de lluvia (diciembre - marzo) y disminuye en época seca (abril - noviembre).

En el análisis de la escorrentía superficial, se aprecia que los tres ecosistemas tienen comportamientos similares entre el 24 y $25 \%$ (Tabla 2). En el caso del bofedal, una vez que el agua subterránea alcanza el nivel superficial del suelo, la lluvia adicional se convertirá en escorrentía (USGS, 2016; Hasan \& Khan 2019); mientras que en el pajonal y bosque de Polylepis, la escorrentía está fundamentalmente controlada por la pendiente y las características del suelo donde se desarrolla.

El servicio de regulación hídrica en el ecosistema

Una mirada completa de las diversas coberturas estudiadas permite apreciar las entradas, salidas y el almacenamiento de agua en los ecosistemas estudiados. 
Los resultados del balance hídrico muestran que la evapotranspiración y la escorrentía son los procesos más importantes de salida de agua del ecosistema. Respecto a la regulación hídrica, se aprecia que el pajonal contribuye con $194 \mathrm{~mm} / \mathrm{año}$, o $15.1 \%$, de toda la lluvia que cae en la cuenca; el bofedal contribuye con 211 mm/año, o 16.4\%, y el bosque de Polylepis con 198 mm/año, o $15.4 \%$ (Tabla 2). Comparativamente, estos resultados serían ligeramente superiores a los hallados por Ochoa-Tocachi et al. (2016) quienes obtuvieron un balance hídrico de $173 \mathrm{~mm}$ por año, en un ambiente análogo de puna húmeda de la zona central del Perú (Huamantanga / Departamento de Lima). Los autores consideran que tales valores son bajos debido al sobrepastoreo en los ecosistemas de puna.

Asimismo, analizando el proceso de regulación hídrica (recarga) durante un año hidrológico, se aprecia que dicho proceso inicia en diciembre y termina en abril, con el inicio y fin de la temporada de lluvias, respectivamente (Figura 5). También se observa que la recarga es un proceso dinámico que va de la mano del comportamiento de la precipitación y las características biofísicas de los ecosistemas: suelo y vegetación. Sin embargo, en el bofedal, el proceso de recarga culmina en febrero. En el bofedal, debido a que el nivel freático se encuentra cerca de la superficie del suelo, cuando el ecosistema se satura pierde la capacidad de infiltración de agua, pues las lluvias posteriores se convertirán en escorrentía. Este proceso está explicado por Hayashi \& Van der Kamp (2000) y Hasan \& Khan (2019), quienes señalan que este comportamiento se observa en aquellos ecosistemas donde el nivel freático se encuentra en la superficie del suelo o muy cerca de ella. A pesar de que en el bofedal el proceso de recarga concluye antes que termine el período de lluvias, se observa que, durante un año hidrológico, tiene un mejor desempeño $(210 \mathrm{~mm})$ que los otros ecosistemas (Figuras 5 y 6). Este hecho se explica por las características del suelo, como la porosidad por la abundancia de turba y la reducida pendiente que reduce la escorrentía superficial y facilita la infiltración.

Extrapolando estos resultados a toda la microcuenca de estudio, se obtiene información adicional sobre la relevancia de cada ecosistema en el proceso de regulación (Tabla 3). Así, en el pajonal se regula el $80 \%$ del agua que queda como aporte para la recarga de los acuíferos, en el bofedal el $17 \%$ y en el bosque de Polylepis el 3\%. De manera conjunta y en términos de volumen de agua, se lograría regular un total de $1417000 \mathrm{~m}^{3}$ durante un año hidrológico (Tabla 3). Este volumen estimado, frente a lo observado como $Q_{\text {Res }}$ (Tabla 4 ), permite apreciar que sus resultados son aproximadamente iguales; es decir: $1417000 \mathrm{~m}^{3} \cong 1516000 \mathrm{~m}^{3}$. Al nivel estacional, que representa la resolución más gruesa para estudiar la regulación hídrica, también se observa caudales similares en la época de estiaje (mayo - noviembre) y en la época de lluvia (diciembre - abril) entre caudales modelados y caudales observados. Estiaje: $410415 \cong$ 448 385; lluvia: $1006776 \cong 1067244$. Este resultado valida parcialmente la calibración específica del modelo Hydrobal para estudiar las contribuciones de diferentes ecosistemas altoandinos en el servicio de regulación hídrica, pero será necesario continuar con el recojo de información para reajustar y corroborar los cálculos.

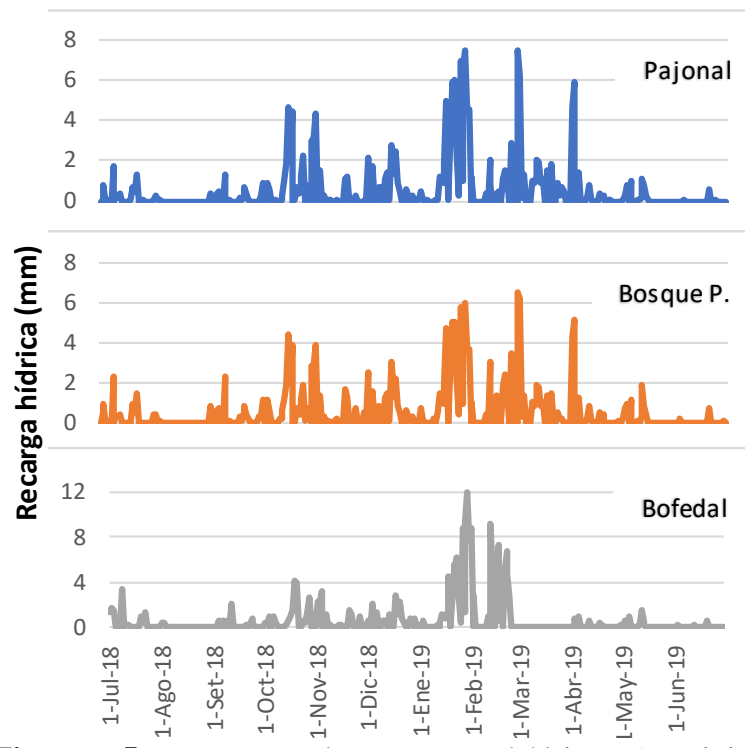

Figura 5. Proceso de recarga hídrica (servicio ecosistémico de regulación) en los ecosistemas estudiados (pajonal de puna húmeda, bofedal y bosque de Polylepis) durante un año hidrológico.

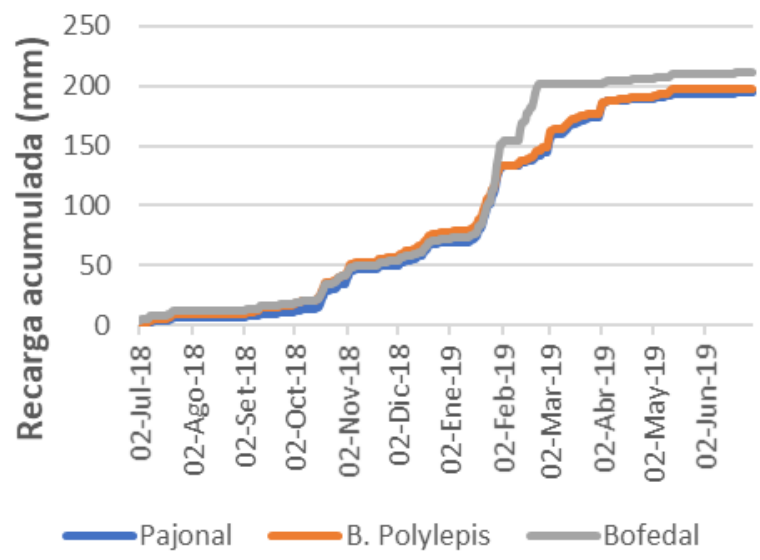

Figura 6. Proceso de infiltración y provisión del servicio ecosistémico de regulación hídrica acumulada en los ecosistemas estudiados: pajonal de puna húmeda, bofedal y bosque de Polylepis.

A partir de los resultados se resaltan dos aspectos centrales en el servicio ecosistémico de regulación hídrica en la unidad hidrográfica de Rontoccocha: su capacidad por unidad de área y a nivel de toda la microcuenca estudiada. Por unidad de área existen diferencias menores entre los tres ecosistemas 
Julio - Diciembre 2021

estudiados; a pesar de ello, el bofedal muestra un ligero mejor desempeño que el bosque de Polylepis y éste mejor que el pajonal. Mientras que, a nivel de toda la unidad hidrográfica, el pajonal es el que más aporta a la regulación con el $79.4 \%$. Algunas de las razones que explican estas contribuciones se debe a las características físicas del entorno; pero también a las características morfológicas, fisiológicas y ecológicas particulares de las plantas de montaña como: ser de tamaño pequeño, con superficie foliar pequeña (LAI), raíces poco profundas, pero bien desarrolladas (Tranquillini, 1964; Billings, 1974; Körner et al., 1989; Körner, 2003), que influyen en los parámetros hidrológicos (Bellot \& Chirino, 2013; Touhami, 2014).

Así mismo, las adaptaciones de las plantas de montaña tienen ventajas para el balance hídrico. En época seca, dichas plantas entran en latencia vegetativa, disminuyen su respiración y su capacidad fotosintética, lo que les permite reducir la pérdida de agua en comparación con plantas que se desarrollan en bajas altitudes (Billings, 1974; Körner, 2003). Por ejemplo, las raíces cortas (pero bien desarrolladas) limitan su alcance a los niveles más profundos de agua y, con ello, la capacidad de extraer y expulsar agua al ambiente mediante la evapotranspiración. En contraste, las raíces extensas de los árboles, a medida que el suelo se seca, extrae agua de las capas más profundas para mantener sus funciones vitales.

A pesar de que la investigación muestra los resultados de forma individual para cada ecosistema, ello se realizó con fines comparativos y avanzar en su entendimiento. En la realidad, éstos se interrelacionan, son interdependientes y funcionalmente están conectados entre sí. Por ejemplo, las interacciones entre ecosistemas se pueden apreciar en los bofedales y lagunas. Estos al estar ubicados sobre terrenos bajos y depresiones naturales, reciben la afluencia de agua subterránea provenientes de la recarga o infiltración que se da en los ecosistemas ubicados cuenca arriba (USGS, 2016) como los pajonales. Así mismo, los bofedales y las lagunas impiden el drenaje rápido del agua de la cuenca y contribuyen en su almacenamiento (Hayashi \& Van der Kamp, 2000; Hasan \& Khan, 2019). Visto así, no se puede gestionar los diferentes elementos de la cuenca de forma aislada, se requiere de un manejo integral. Así mismo, en cuencas donde existen presas de agua, estas interacciones también refuerzan la idea de complementariedad que debe existir entre infraestructura gris $o$ física $\mathrm{e}$ infraestructura natural que, combinadas, brindan soluciones más eficientes y sostenibles (Cohen et al., 2016).

Finalmente, es necesario señalar algunas limitaciones del trabajo. Primero, el estudio no presenta resultados sobre el aporte hídrico por efecto de la recuperación de los ecosistemas; es decir, modela la situación actual. Por ello, se tendrá que continuar con las investigaciones que permitan medir la contribución hídrica adicional de las acciones de restauración de los ecosistemas andinos. Segundo, también se debe continuar con la validación de la herramienta Hydrobal en la zona andina, dado que originalmente fue desarrollada para regiones semiáridas del mediterráneo; sin embargo, su valor está en su capacidad para determinar el efecto de los tipos de vegetación en el balance hídrico y la recarga del acuífero (Bellot \& Chirino, 2013). Para ambos casos, se deberá continuar con las acciones de monitoreo de las cuencas andinas que permitan enriquecer la escasa data climática, de cobertura vegetal, ecológica, hidrológica, edafológica y otros. Sobre esa base, también se podría robustecer el modelo al tener una mayor serie de tiempo.

\section{Conclusiones}

La investigación muestra cómo los ecosistemas altoandinos, ubicados por encima de los $3900 \mathrm{msnm}$, contribuyen al servicio ecosistémico de regulación hídrica. Por tanto, la regulación en los tres ecosistemas evaluados (pajonal, bofedal y bosque de Polylepis) no muestra diferencias mayores por unidad de área; sin embargo, a nivel de la cuenca, se aprecia que el pajonal andino es el ecosistema que más aporta a la regulación hídrica $(80 \%)$ debido a su extensa cobertura en el territorio. En tal sentido, esta investigación contribuye al entendimiento sobre la importancia de tales ecosistemas para la provisión de agua y, a partir de ello, también brinda elementos para mejorar políticas y prioridades de inversión relacionadas a la siembra de agua y afianzamiento hídrico.

\section{Agradecimientos}

Especial agradecimiento al FONDECYT, iniciativa del CONCYTEC Perú, por financiar la presente investigación en el marco del Doctorado en Economía de los Recursos Naturales y Desarrollo Sustentable de la Universidad Nacional Agraria la Molina (Convenio $\mathrm{N}^{\circ}$ 200-2015-FONDECYT).

\section{Literatura citada}

Bellot J. \& Chirino E. 2013. Hydrobal: An eco-hydrological modelling approach for assessing water balances in different vegetation types in semi-arid areas. Ecological Modelling, 266: 30-41.

https://doi.org/10.1016/j.ecolmodel.2013.07.002.

Billings W.D. 1974. Adaptations and origins of alpine plants. Arctic and Alpine Research, 6(2): 129-142. https://www.tandfonline.com/doi/abs/10.1080/00040851 .1974 .12003769 .

Bonnesoeur V., Locatelli B., Ochoa-Tocachi B., Vanacker V., Mao Z., Stokes A. \& Mathez-Stiefel S. 2019. Impacts of forests and forestation on hydrological services in the Andes: a systematic review. Forest Ecology and Management, 433: 569-

584. https://doi.org/10.1016/j.foreco.2018.11.033. 
Brown C., Reyers B., Ingwall-King L., Mapendembe A., Nel J., O'Farrell P., Dixon M. \& Bowles-Newark N. 2014. Measuring ecosystem services: guidance developing ecosystem services indicators. Cambridge, UK: UNEPWCMC.

https://www.unepwcmc.org/system/dataset_file_fields/files/000/000/303/o riginal/1850_ESI_Guidance_A4_WEB.pdf?1424707843

Buytaert W., Célleri R., De Bièvre B., Cisneros F., Wyseure G., Deckers J. \& Hofstede R. 2006. Human impact on the hydrology of the Andean páramos. Earth-Science Reviews, 79(1-2): 53-72. https://doi.org/10.1016/j.earscirev.2006.06.002.

Cohen-Shacham E., Walters G., Janzen C. \& Maginnis S. 2016. Nature-based Solutions to address global societal challenges. Gland, Switzerland: UICN. https://doi.org/10.2305/IUCN.CH.2016.13.en.

ESA. 2018. Copernicus open access hub. ESA (The European Space Agency) Consultado en junio de 2019 de: https://scihub.copernicus.eu/.

GRA. 2010. Zonificación ecológica y económica de la región Apurímac - ZEE. GRA (Gobierno Regional de Apurímac). Abancay, Apurímac, Perú.

GRA. 2013. Hidrología de la cuenca del río Mariño. Un estudio definitivo del proyecto de gestión integral de la microcuenca Mariño - Apurímac. U.E. Pro Desarrollo Apurímac. GRA (Gobierno Regional de Apurímac). Abancay, Perú.

Hasan H. \& Khan A. 2019. Groundwater and Surface Water Interaction (Chapter 14). In: Venkatramanan S., Prasanna M.V. \& Chung S.-Y. (eds). GIS and Geostatistical Techniques for Groundwater Science. 197-207. Amsterdam, Netherlands. https://doi.org/10.1016/B9780-12-815413-7.00014-6.

Hayashi M. \& Van der Kamp G. 2000. Simple equation to present the volume-area-depth relations of shallow wetlands in small topographic depressions. Journal of Hydrology, 237(1-2): 74-85. https://doi.org/10.1016/S0022-1694(00)00300-0.

Körner C., Neumayer M., Pelaez S. \& Smeets-Scheel A. 1989. Functional Morphology of Mountain Plants. Flora, 182(5-6): 353-383. https://doi.org/10.1016/S03672530(17)30426-7.

Körner C. 2003. Alpine Plant Life. Functional Plant Ecology of High Mountain Ecosystems. 2 ed. Springer. Basilea, Suiza. https://doi.org/10.1007/978-3-642-18970-8.

León F. 2016. Inversión en infraestructura natural: haciendo sostenibles las inversiones en infraestructura física. Cooperación Alemana al Desarrollo - Agencia de la GIZ en el Perú. Lima.

Llambí L., Soto A., Célleri R., De Bievre B., Ochoa B. \& Borja P. 2012. Páramos Andinos: Ecología, hidrología y suelos de páramos. Proyecto Páramo Andino: CONDESAN, Instituto Humboldt (Colombia), EcoCiencia, Instituto de Montaña, ICAE (Instituto de Ciencias Ambientales y Ecológicas), GEF, UNEP, etc.). https://www.portalces.org/sites/default/files/references/0 40_LLamb\%C3\%AD\%20et\%20al.\%202012.PPEcologia\%20Hidrologia\%20y\%20Suelos\%20de\%20Par amos_0.pdf.

Lynn Hood J., W Roy J. \& Hayashi M. 2006. Importance of ground water in the water balance of alpine headwater lake. Geophysical Research Letters, 33(13): 1-5. https://doi.org/10.1029/2006GL026611.
MINAM. 2019. RM No 178-2019-MINAM: Lineamientos para la formulación de proyectos de inversión en las tipologías de ecosistemas, especies y apoyo al uso sostenible de la biodiversidad. El Peruano (Normas Legales), 36(14969): 7-8. Domingo 9 de Junio de 2019. MINAM (Ministerio del Ambiente). Lima / Perú. https://busquedas.elperuano.pe/download/url/apruebanlos-lineamientos-para-la-formulacion-de-proyectosresolucion-ministerial-n-178-2019-minam-1777515-1. https://cdn.www.gob.pe/uploads/document/file/319848/ RM_N_178-2019.pdf.

Ochoa-Tocachi B., Buytaert W., De Bièbre B., Célleri R., Crespo P., Villacís M., Llerena CA., Acosta L., Villazón M., Guallpa M., Gil-Ríos J., Fuente P., Olaya D., Viñas P., Rojas G. \& Arias S. 2016. Impacts of land use on the hydrological response of tropical Andean catchments. Hydrological Processes, 30(22): 4074-4089. https://doi.org/10.1002/hyp.10980.

Somers L. \& McKenzie J. 2020. A review of groundwater in high mountain environments. Wires Water, 7(6): e1475. https://doi.org/10.1002/wat2.1475.

Sprenger F. 1978. Determination of direct runoff with the curve number method in the coastal area of Tanzania/East Africa. Wasser Boden, I: 13-16.

Touhami I. 2014. Estimación del balance hídrico y de la recarga en el acuífero Ventós - Castellar (SE España). Efectos del cambio climático. Tesis Ph.D . España, Alicante, Universidad de Alicante. URI: http://hdl.handle.net/10045/41923.

Tranquillini W. 1964. The physiology of plants at high altitudes. Annual Review of Plant Physioogyl, 15: 345362. https://doi.org/10.1146/annurev.pp.15.060164.002021.

USGS. 2016. Natural Processes of ground-water and surfacewater interaction. In: Winter T.C., Harvey J.W., Franke O.L. \& Alley W.M. Ground Water and Surface Water a Single Resource (https://pubs.usgs.gov/circ/circ1139/). USGS (United States Geological Survey), Circular 1139. Consultado en enero de 2020 de: https://pubs.usgs.gov/circ/circ1139/htdocs/natural_proce sses_of_ground.htm.

USDA. 2016. Natural Resources Conservation Service. Soil Water Characteristics. USDA (United States Department of Agriculture). Consultado en abril de 2020 de: https://www.nrcs.usda.gov/.

Valencia-Leguizamón J. \& Tobón C. 2017. Influencia de la vegetación en el funcionamiento hidrológico de cuencas de humedales de alta montaña tropical. Ecosistemas, 26(2): 10-17. DOI: 10.7818/ECOS.2017.26-2.02.

Vallet A., Locatelli B., Levrel H., Dendoncker N., Barnaud C. \& Quispe Y. 2019. Linking equity, power and stakeholders' roles in relation to ecosystem services. Ecology and Society, 24(2): Art. 14. https://doi.org/10.5751/ES-10904-240214.

Vallet A. Locatelli B. \& Pramova E. 2020. Servicios ecosistémicos y equidad social: ¿quién controla, quién se beneficia y quién pierde? Infobrief, (311): 1-8. Traducción del No. 307 de November 2020. https://doi.org/10.17528/cifor/007850.

Viviroli D., Durr H., Messerli B., Meybeck M. \& Weingartner R. 2007. Mountains of the world, water towers for humanity: Typology, mapping, and global significance. Water Resource Research, 43(7): 1-13. https://doi.org/10.1029/2006WR005653. 
Tabla 1. Principales características del suelo en los ecosistemas estudiados e ingresados en el modelo ecohidrológico Hydrobal.

\begin{tabular}{|c|c|c|c|c|}
\hline \multirow[t]{2}{*}{ Variable } & Símbolo & Bofedal & Pajonal & Bosque de Polylepis \\
\hline & & \multicolumn{3}{|c|}{ Valor Promedio (Desviación Estándar) [Min, Max] } \\
\hline Humedad & Hobs. (\%) & $76.2(8.27)$ & $35.3(7.16)$ & $50.3(3.51)$ \\
\hline observada & & {$[64.0-91.4]$} & {$[26.4-50.0]$} & {$[47.0-54.0]$} \\
\hline Capacidad & $\mathrm{CC}(\%)$ & $28.7(3.50)$ & $29.4(3.63)$ & $27.4(1.62)$ \\
\hline campo & & {$[23.2-33.5]$} & {$[21.1-35.0]$} & {$[25.7-28.9]$} \\
\hline Punto de marchitez & $\operatorname{Pmp}(\%)$ & $16.4(2.89)$ & $11.9(2.42)$ & $15.7(1.02)$ \\
\hline permanente & & {$[11.1-21.3]$} & {$[7.8-16.4]$} & {$[14.5-16.6]$} \\
\hline Porosidad & Ptotal (\%) & $72.4(7.00)$ & $48.3(4.49)$ & $56.4(2.47)$ \\
\hline & & {$[61.2-83.4]$} & {$[41.0-58.2]$} & {$[54.3-59.1]$} \\
\hline Humedad inicial & $\mathrm{Hi}(\%)$ & $16.4(2.89)$ & $11.89(2.42)$ & $15.7(1.02)$ \\
\hline & & {$[11.1-21.3]$} & {$[7.80-16.4]$} & {$[14.6-16.6]$} \\
\hline$\%$ de cobertura del & Cover $(\%)$ & $93.7(6.44)$ & $82.0(15.4)$ & $68.0(9.13)$ \\
\hline suelo & & {$[80-100]$} & {$[50-100]$} & {$[60-85]$} \\
\hline Lluvia neta* & Ln. (\%) & 99 & 99 & 87 \\
\hline $\begin{array}{l}\text { Factor k de evapo- } \\
\text { transpiración }\end{array}$ & $\mathrm{K}(\sin )$ & 0.07 & 0.06 & 0.06 \\
\hline Textura & $\mathrm{Tx}$ & $\begin{array}{l}\text { Franco limoso, franco } \\
\text { arenoso orgánico }\end{array}$ & $\begin{array}{l}\text { Franco arcillo arenoso, } \\
\text { franco limoso }\end{array}$ & Franco arenoso \\
\hline Prof. nivel freático & Prof $(\mathrm{cm})$ & $\begin{array}{c}21(11.99) \\
{[-65-0]}\end{array}$ & & \\
\hline $\begin{array}{l}\text { Número de curva, } \\
\text { época seca }\end{array}$ & $\mathrm{CN}(\sin )$ & - & 47.1 & 45.0 \\
\hline $\begin{array}{l}\text { Número de curva, } \\
\text { época lluviosa }\end{array}$ & $\mathrm{CN}(\sin )$ & - & 67.0 & 67.0 \\
\hline
\end{tabular}

Tabla 2. Resultados del modelo Hydrobal para cada ecosistema en estudio (en mm y, entre paréntesis, en $\%$ del total de precipitación).

\begin{tabular}{lcccccc}
\hline Ecosistema & Pp & Int. & Pp neta & Esc. & Eta real & Recarga \\
\hline Pajonal & 1290 & 51.5 & 1240 & 324 & 698 & 194 \\
& $(100 \%)$ & $(4.0 \%)$ & $(96.0 \%)$ & $(25.1 \%)$ & $(54.2 \%)$ & $(15.1 \%)$ \\
Bofedal & 1290 & 25.8 & 1260 & 320 & 765 & 211 \\
& $(100 \%)$ & $(2.0 \%)$ & $(98.0 \%)$ & $(24.9 \%)$ & $(59.4 \%)$ & $(16.4 \%)$ \\
Bosque & 1290 & 122 & 1170 & 311 & 696 & 198 \\
Polylepis & $(100 \%)$ & $(9.5 \%)$ & $(90.5 \%)$ & $(24.2 \%)$ & $(54.1 \%)$ & $(15.4 \%)$ \\
\hline
\end{tabular}

Tabla 3. Servicio de regulación hídrica de los ecosistemas, extrapolado a toda el área de la unidad hidrográfica de Rontoccocha.

\begin{tabular}{lrrrr}
\hline \multicolumn{1}{c}{ Ecosistemas } & $\begin{array}{c}\text { Área } \\
\text { (ha) }\end{array}$ & \multicolumn{1}{c}{$\begin{array}{c}\text { Regulación } \\
(\mathbf{m m})\end{array}$} & $\begin{array}{c}\text { Regulación } \\
\text { total (m3) }\end{array}$ & $\%$ \\
\hline Pajonal & 585 & 194 & 1136000.00 & 80 \\
Bofedal & 121 & 210 & 240000.00 & 17 \\
Bosque de Polylepis & 19.3 & 198 & 40700.00 & 3 \\
& & Total & 1417000.00 & 100 \\
\hline
\end{tabular}


Tabla 4. Balance hídrico de la laguna Rontoccocha y estimación del volumen de agua subterránea $Q_{\text {Res }}$ para validar el modelamiento de Hydrobal. Q in (volumen de ingreso de agua superficial), h (altura del nivel de almacenamiento de agua en la laguna), $\mathrm{P}$ (precipitación), E (Evaporación) $\Delta \mathrm{V} / \Delta \mathrm{t}$ (cambio en el almacenamiento).

\begin{tabular}{|c|c|c|c|c|c|c|c|}
\hline Mes & $\begin{array}{c}\mathbf{h} \\
(\mathrm{m})\end{array}$ & $\begin{array}{c}\mathbf{Q}_{\text {in }} \\
(\mathrm{m} 3)\end{array}$ & $\begin{array}{l}\text { Qout } \\
\text { (m3) }\end{array}$ & $\begin{array}{c}\mathbf{P} \\
(\mathrm{m} 3)\end{array}$ & $\begin{array}{c}\mathbf{E} \\
(\mathrm{m} 3)\end{array}$ & $\begin{array}{c}\Delta \mathbf{V} / \Delta \mathbf{t} \\
(\mathrm{m} 3)\end{array}$ & $\begin{array}{l}\text { QRes } \\
\text { (m3) }\end{array}$ \\
\hline Mayo & 2.3 & 97146 & 166436 & 2070 & 8850 & 30000.0 & 106070 \\
\hline Junio & 2.5 & 80430 & 15033 & 1650 & 9750 & -36000.0 & 6703 \\
\hline Julio & 2.26 & 70040 & 118867 & 1440 & 10800 & 18000.0 & 76187 \\
\hline Agosto & 2.38 & 60813 & 118867 & 4200 & 11550 & -63000.0 & 2404 \\
\hline Setiembre & 1.96 & 52021 & 115033 & 2400 & 10800 & 21000.0 & 92412 \\
\hline Octubre & 2.1 & 74674 & 113966 & 7920 & 11100 & 84000.0 & 126472 \\
\hline Noviembre & 2.66 & 87273 & 142690 & 7830 & 10050 & -19500.0 & 38137 \\
\hline Diciembre & 2.53 & 103842 & 246279 & 37830 & 8250 & 25500.0 & 138357 \\
\hline Enero & 2.7 & 281902 & 506084 & 40500 & 6750 & 0.0 & 190432 \\
\hline Febrero & 2.7 & 285137 & 588315 & 31380 & 5550 & 0.0 & 277348 \\
\hline Marzo & 2.7 & 277750 & 635316 & 28050 & 6300 & -45000.0 & 290816 \\
\hline Abril & 2.4 & 167184 & 396965 & 6690 & 7200 & -60000.0 & 170291 \\
\hline
\end{tabular}

\section{Leyenda}

\section{$\sim$ Red hídrica \\ C Limite microcuenca}

\begin{tabular}{|c|c|c|c|}
\hline Cobertura & $\begin{array}{l}\text { Área } \\
\text { (ha) }\end{array}$ & $\%$ & Color \\
\hline Pajonal de puna húmeda & 584.7 & 66.9 & \\
\hline Bofedal & 121.1 & 13.9 & \\
\hline Bosque de Polylepis & 19.3 & 2.2 & \\
\hline Lagunas altoandinas & 35.0 & 4.0 & \\
\hline Afloramientos rocosos & 114.4 & 13.1 & \\
\hline & 17.1 & 10.1 & \\
\hline
\end{tabular}
Total

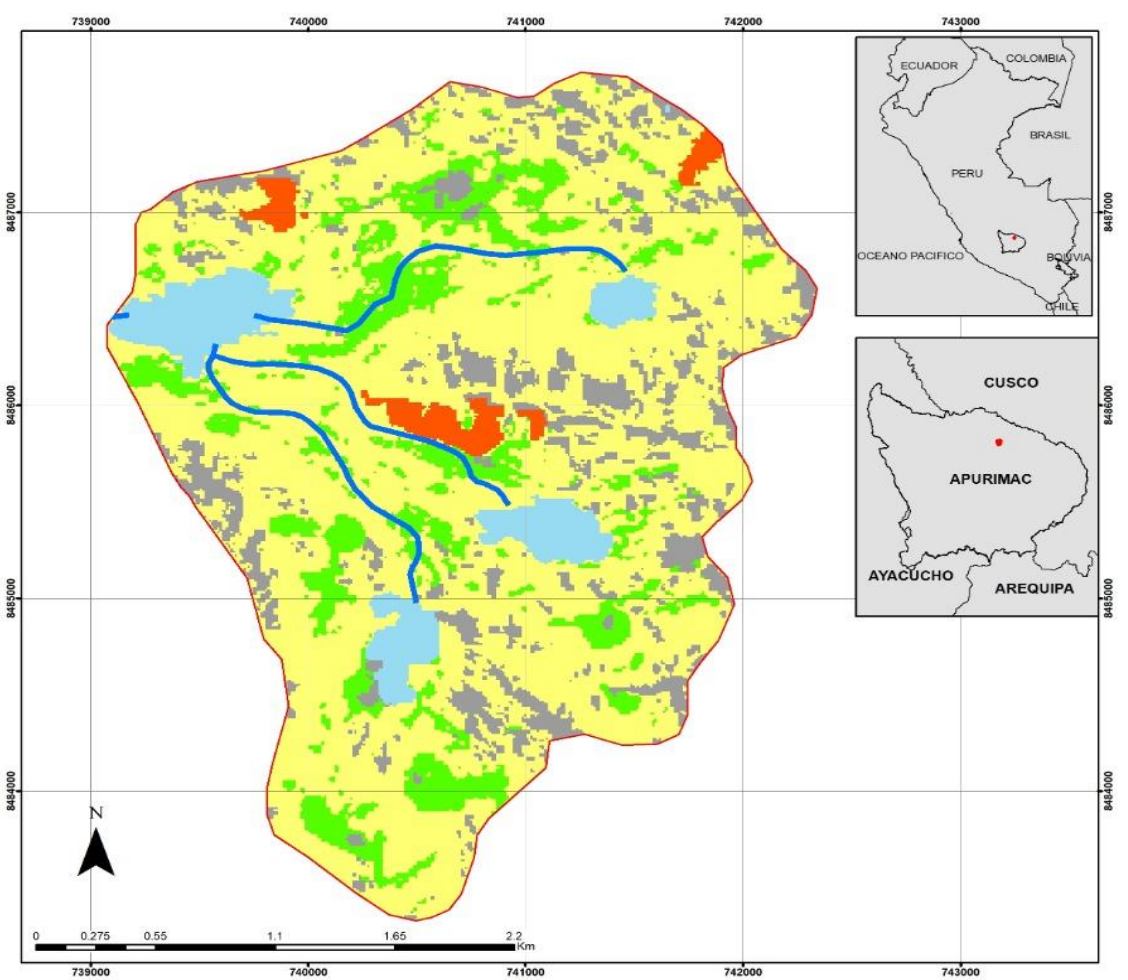

Figura 1. Ámbito de estudio: unidad hidrográfica Rontoccocha.

\footnotetext{
${ }^{1}$ Universidad Nacional Agraria la Molina, Av. La Molina s/n, 15024 La Molina / Lima / Perú.

2 Superintendencia Nacional de Servicios de Saneamiento (SUNASS), Av. Garcilaso de la Vega 168, 03001 Abancay / Perú.

${ }^{3}$ Autor de correspondencia: ronalcervantes@gmail.com.

4 jmsanchez@lamolina.edu.pe.

5 jalegre@lamolina.edu.pe.

${ }^{6}$ ericrendon@lamolina.edu.pe.
} 
${ }^{7}$ Programa Bosques Andinos, HELVETAS Swiss Intercooperation, Av. Ricardo Palma 857, Miraflores, 450345 Lima / Perú. Asociación para la Conservación y Estudio de Montañas Andinas-Amazónicas (ACEMAA), Av. Santa Martha, San Jerónimo / Cusco / Perú. Iniciativa Regional de Monitoreo Hidrológico de Ecosistemas Andinos (iMHEA), Lima / Perú. EClim Research Group / University of Zurich, Switzerland. jbaiker@gmail.com.

${ }^{8}$ Centro para la Investigación Forestal Internacional (CIFOR, por sus siglas en inglés), Av. La Molina 1895, 15024 La Molina / Lima / Perú. Centro de Cooperación Internacional en Investigación Agronómica para el Desarrollo (CIRAD, por sus siglas en francés), Universidad de Montpellier, 34398 Montpellier / Francia. bruno.locarelli@cirad.fr.

${ }^{9}$ Consorcio para el Desarrollo de la Ecorregión Andina (CONDESAN), CA: Las Codornices 253, 15047 Surquillo / Lima / Perú. vivien.bonnesoeur@inshcondesan.org. 